\title{
Envolvimento Ocular nas Doenças Reumáticas
}

\section{Ocular Involvement in Patients with Rheumatic Diseases}

\author{
Seleção de artigos e comentários feitos por \\ Ivânio Alves Pereira ${ }^{(1)}$
}

\begin{abstract}
As manifestações oculares que podem ocorrer em pacientes com doenças reumáticas e algumas das opções terapêuticas atuais têm sido objeto de publicações científicas. Vale lembrar que a incidência de doenças sistêmicas em uveíte chega a 40\% e que em grande parte as uveítes são causadas pelas doenças reumáticas. Medicações que incluem corticosteróides, imunossupressores e agentes biológicos, utilizadas freqüientemente em doença inflamatória ocular severa, não são de uso continuado pelos serviços de oftalmologia, o que faz com que inúmeras vezes reumatologistas sejam solicitados para investigar, acompanhar, prescrever medicamentos ou orientar condutas em situações de doença sistêmica.
\end{abstract}

Joseph A, Raj D, Dua HS, Powel PT, Lanyon PC, Powell RJ: Infliximab in the treatment of refractory posterior uveitis (Infliximab no tratamento da uveíte posterior refratária). Ophtalmology 110: 1449-53, 2003. Instituição: Queen's medical center, Nothinghan, Reino Unido.

Os autores analisaram os resultados do uso do infliximab em 5 pacientes com uveíte posterior grave e risco de perda de visão, refratários ao uso de outras drogas imunossupressoras. Dos 5 pacientes, 3 tinham síndrome de Behçet e 2 tinham uveíte posterior idiopática. A resposta terapêutica foi avaliada através do escore de oftalmoscopia indireta binocular, da presença de vasculite de retina e acuidade visual. Após duas semanas da primeira aplicação do infliximab, 4 dos 5 pacientes mostraram melhora marcante na turvação do vítreo e acuidade visual. Após um seguimento de seis meses, estes 4 pacientes alcançaram remissão da uveíte posterior e puderam ser retirados de qualquer outro tratamento imunossupressor. Três pacientes necessitaram novas infusões de infliximab. Um dos pacientes desenvolveu tuberculose ocular e sistêmica, que respondeu ao tratamento com tuberculostáticos. Os autores assim concluíram que infliximab é realmente efetivo no tratamento da uveíte posterior refratária, mas que estes pacientes devem ser investigados para tuberculose antes do tratamento e vigiados após as infusões do medicamento.

Apesar do reduzido número de pacientes, a gravidade do problema e a ótima resposta a esta nova terapêutica alertam para a sua utilização em situações de risco de perda de visão.

Kidd D, Burton B, Plant GT, Grahan EM: Chronic relapsing inflammatory optic neuropathy ( CRION) (Neuropatia óptica inflamatória recorrente crônica). Brain 126: 276 84, 2003. Instituição: St Thomas Hospital, Londres.

Os autores descrevem as características clínicas e a história natural de uma forma de neuropatia óptica inflamatória recorrente, freqüentemente bilateral e dolorosa, caracterizada por recidivas e remissões. A ressonância magnética de crânio é normal e a ressonância dos nervos ópticos freqüentemente mostram aumento da intensidade de sinal. Os sinais e sintomas

1. Médico assistente do serviço de reumatologia do Hospital Universitário da Universidade Federal de Santa Catarina (UFSC), Florianópolis, SC. 
respondem bem aos corticosteróides, embora imunossupressores a longo prazo freqüentemente sejam necessários. Os autores assim descrevem uma série de 15 pacientes em 10 anos, com neuropatia óptica subaguda que responderam imediatamente aos corticosteróides, mas recidivaram com a sua retirada. O grau de perda de visão nos casos descritos foi mais severo que nos casos de neurite óptica desmielinizante. O envolvimento de ambos os nervos ópticos foi comum e geralmente seqüencial. $\mathrm{O}$ quadro dos pacientes assemelha-se a uma neurosarcoidose localizada, no qual inexistem evidências de lesões granulomatosas disseminadas em outros órgãos. É importante reconhecer que os casos descritos pelos autores também diferem das neuropatias ópticas desmielinizantes, já que não há alterações na ressonância magnética de crânio, tampouco alterações na síntese de imunoglobulinas intra-tecais. No caso de neuropatia óptica inflamatória recorrente, imunossupressores foram utilizados em situações de dependência aos corticosteróides ou de refratariedade.

Rechia FM, Brown GC: Systemic disorders associated with retinal vascular occlusion (Doenças sistêmicas associadas com oclusão vascular da retina). Cur Opin Opthalmol 11: 462-7, 2000. Instituição: Serviço de retina Wills Eye Hospital, Philadelfia.

Oclusões das artérias da retina e da circulação venosa são causas comuns de piora da visão, podendo acometer todos os grupos etários. Entre os fatores associados, encontram-se as doenças cérebro-vasculares e as doenças cardiovasculares, que podem, inclusive, requerer tratamento sistêmico. A obstrução venosa da retina pode ser a apresentação clínica de hipertensão arterial sistêmica, diabetes melito e doença cardiovascular. Os autores revisaram as patologias sistêmicas associadas à oclusão da artéria da retina. As oclusões da artéria central da retina comumente se manifestam por declínio agudo e irreversível na acuidade visual. A retina pode ter aparência normal, mas comumente está pálida e edemaciada, com aspecto opacificado. Êmbolos na retina podem ser visíveis e originam-se tipicamente de placas ateroscleróticas ulceradas, de trombos dentro de artérias carótidas internas ou mesmo de válvulas cardíacas. Quase metade dos pacientes com oclusão da artéria da retina demonstram alterações no ecocardiograma, que incluem endocardite bacteriana, doença valvular cardíaca, infarto do miocárdio, prótese valvar, cardiopatia congênita e fibrilação atrial. Relatos de casos de deficiência de proteínas anticoagulantes endógenas como anti-trombina III, proteína $\mathrm{C}$, proteína $\mathrm{S}$ e mutação do fator $\mathrm{V}$ de Leiden podem estar presentes em pacientes com oclusão da artéria ou veia da retina. Da mesma forma, relatos de síndrome antifosfolipídica primária e oclusões arteriolares ou venosa da retina bilaterais têm sido publicados. Causas inflamatórias sistêmicas de oclusões arteriais da retina incluem o lúpus eritematoso sistêmico, granulomatose de Wegener e displasia fibromuscular. Homocisteína é considerado atualmente um fator de risco independente para oclusão arterial ou venosa da retina. Os autores assim discutem que todos os pacientes com oclusão da artéria central da retina ou ramos da artéria da retina devem ser submetidos a ecocardiografia e ultra-sonografia de carótidas. Pacientes com oclusão da veia central da retina ou de ramos venosos da retina devem ser investigados para a presença de hipertensão arterial sistêmica, diabetes melito, anormalidades lipídicas e elevações da homocisteína. Uma pesquisa para hipercoagulabilidade, incluindo anticorpos antifosfolipídicos, deve ser realizada nos pacientes com história de eventos trombóticos.

Goldstein DA, Fontanilha FA, Kaul S, Sahin O, Tessler HH: Long-term follow-up of patients treated with shortterm high-dose chlorambucil for sight-threatening ocular inflammation (Seguimento a longo prazo dos pacientes tratados com altas doses de clorambucil por curto prazo, nas inflamações oculares com risco de perda de visão). Ophthalmology 109: 370-7, 2002. Instituição: Universidade de Illinois.

Os autores realizaram estudo com o objetivo de avaliar a eficácia e os efeitos adversos de altas doses de clorambucil por curto período em pacientes com uveíte grave e risco de perda de visão. Foram tratados 53 pacientes na enfermaria de oftalmologia da Universidade de Illinois e também no consultório particular de um dos autores. O desenho do estudo foi retrospectivo, não comparativo e de intervenção. Os pacientes foram avaliados através da acuidade visual, 
grau de inflamação ocular e o do desenvolvimento de efeitos colaterais. A indicação para o uso de clorambucil nesta série incluiu uveíte não infecciosa severa com risco de perda de visão, e com pobre resposta prévia ou efeitos intolerantes ao uso de corticosteróides ou ao uso de outros imunossupressores. Perda de visão foi definida pelos autores como envolvimento no segundo olho de um paciente que já apresentava incapacidade visual no primeiro olho, ou doença que comprometia de forma importante a mácula no outro olho, ou doença ocular que causava mau funcionamento dos fotorreceptores difusamente. Cinqüenta e três pacientes foram tratados com clorambucil por pelo menos três semanas, sendo que 39 foram reexaminados e 10 foram incapazes de retornar. Nove destes 10 pacientes que não retornaram foram entrevistados por telefone e um foi contatado por e-mail. Três pacientes foram perdidos de seguimento e 1 faleceu por problema cardíaco três meses após o tratamento com clorambucil. Os diagnósticos mais comuns foram: doença de Behçet (32\%), oftalmia simpática (15\%), esclerite $(11 \%)$, iridociclite severa com edema macular cistóide $(11 \%)$, coroidite serpiginosa $(9 \%)$, vasculite da coróide/retina (8\%), Vogt-Koyanagi-Harada (4\%), uveíte intermediária $(2 \%)$, retinocoroidopatia de birdshot $(2 \%)$, xantogranuloma juvenil (2\%) e retinopatia auto-imune(2\%). A duração média do uso do clorambucil foi 16 semanas. A dose média máxima de clorambucil foi $20 \mathrm{mg}$ por dia, com variação de $10 \mathrm{mg}$ a $30 \mathrm{mg}$. Dos 53 pacientes tratados com clorambucil, 41 (77\%) estavam ainda em remissão, sem recorrência da doença na última avaliação e sem necessidade de novas medicações de uso sistêmico, no seguimento médio de 54 meses. Doze pacientes (23\%) tiveram doença recorrente, com necessidade de corticosteróides orais e/ ou outros imunossupressores. Amenorréia secundária desenvolveu em 7 mulheres (26\%), herpes zoster não oftálmico ocorreu em 6 pacientes e 2 pacientes necessitaram transfusões de plaquetas. Nenhum paciente desenvolveu malignidade e 1 paciente foi submetido à esplenectomia por trombocitopenia recorrente, a qual ocorreu após o tratamento, mas não foi considerada secundária ao uso do clorambucil. Do total de pacientes, $47 \%$ apresentaram melhora em pelo menos duas linhas na escala de acuidade visual de Snellen, sendo que 33\% apresentaram melhora em pelo menos três linhas. Seis pacientes (11\%) tiveram uma piora da visão apesar do tratamento com clorambucil, enquanto 8 pacientes $(15 \%)$ não tiveram alterações na acuidade visual. Os autores assim propõem que, em casos de uveíte severa, os pacientes sejam inicialmente tratados com corticosteróides orais; se estes forem insuficientes, imunossupressores como metotrexate, azatioprina ou ciclosporina podem ser ministrados. Em casos de doença severa não responsiva aos corticosteróides ou aos agentes poupadores de corticosteróides, clorambucil deve ser considerado. O seu uso deve ser por curta duração, pois comumente leva à remissão e evita a necessidade de corticoterapia prolongada ou uso prolongado de outro imunossupressor.

Zierhut M, Stübiger N, Aboalchamat W, Jandenberger H, Bialasiewicz A, Engelmann K: Immunnosuppressive therapy with mycophenolate mofetil (cellcept) in treatment of uveitis (Terapia imunossupressora com micofenolato mofetil no tratamento das uveítes). Der Ophtalmologe: Zeitschrift Der Deutschen Ophtalmologischen Gesellschaft 98: 647-51, 2001. Instituição: Universidade Tübingen.

Formas severas de uveíte comumente necessitam ser tratadas com imunossupressão sistêmica. Todas as drogas imunossupressoras disponíveis são conhecidas por sua alta freqüência relativa de efeitos adversos. Micofenolato mofetil, uma droga imunossupressora nova tem mostrado boa eficácia no tratamento dos transplantes de órgãos e no tratamento de muitas doenças auto-imunes. O uso dessa droga em uveíte, como monoterapia ou combinado com corticosteróide, tem mostrado menos efeitos adversos. O objetivo deste estudo multicêntrico foi investigar se a monoterapia com micofenolato mofetil é efetiva nas várias formas de uveíte. Foram tratados 10 pacientes, dos quais 3 tinham uveíte anterior, 2 tinham uveíte intermediária, 4 tinham panuveíte e 1 vasculite de retina. Os pacientes foram tratados através de um protocolo de estudo prospectivo, com um grama de micofenolato mofetil, duas vezes ao dia. A imunossupressão prévia foi descontinuada em consequiência do aparecimento de efeitos adversos ou da ineficácia do tratamento. O tempo de seguimento variou de 1 a 12 meses (média de 4,5 meses). Durante o uso de micofenolato mofetil, 5 dos 10 pacientes utilizaram simultaneamente prednisolona e 1 metotrexate. Dos 10 pacientes, 8 ficaram 
livres de recorrências da uveíte. No início do tratamento com micofenolato mofetil, 1 paciente em uso de ciclosporina A melhorou somente após a troca da ciclosporina para metotrexate. Um paciente com uveíte bilateral recorrente necessitou de corticoterapia complementar para recorrência em um dos olhos. Efeitos adversos foram diarréia em 1 paciente, e outros sintomas gastrintestinais em outro paciente, provocando a interrupção do tratamento em ambos. Os autores assim concluem que o mico- fenolato mofetil cessou a atividade inflamatória ocular em 8 dos 10 pacientes e reduziu de forma drástica a incidência de recorrência, posto que não haviam apresentado boa resposta com outros imunossupressores. Os efeitos adversos foram toleráveis, quando comparados com outros imunossupressores. Os autores alertam para a necessidade de outros estudos com maiores tempo de seguimento e número de pacientes. Apontam para a necessidade de um estudo comparativo com ciclosporina A a longo prazo.

Samson CM, Waheed N, Baltatzis S, Foster S: Methotrexate therapy for chronic noninfectious uveitis. Analysis of a case series of 160 patients (Terapia com metotrexate para uveíte não infecciosa crônica. Análise de uma série de 160 pacientes). Ophtalmology 108: 1134-9, 2001. Instituição: Massachusetts Eye and Ear Infirmary, serviço de imunologia ocular e uveíte, Boston.

Os autores avaliaram a evolução dos pacientes com uveíte não infecciosa crônica, não responsivos ao tratamento com antiinflamatórios e tratados com metotrexate. O estudo incluiu todos os pacientes que usaram metotrexate para uveíte entre 1985 e 1999 naquela instituição. Foram avaliados o grau de controle da inflamação ocular, o efeito poupador de corticosteróide do metotrexate, a acuidade visual e os efeitos adversos do uso do metotrexate. Um total de 160 pacientes foram avaliados, sendo que o controle da inflamação ocular ocorreu em $76,2 \%$, o efeito poupador de corticosteróide ocorreu em $56 \%$ e a acuidade visual melhorou ou foi mantida em 90\% dos pacientes. Efeitos adversos que provocaram a descontinuidade do metotrexate ocorreram em 18\% dos pacientes, porém, havendo efeitos adversos severos em apenas $8,1 \%$ dos pacientes. Não ocorreram morbidade ou mortalidade a longo prazo, secundárias ao uso do metotrexate. Investigação laboratorial foi realizada nos pacientes com hemograma, VDRL, FAN, HLA-B27 e sorologia para toxoplasmose conforme o quadro clínico individual do paciente. A uveíte foi classificada de acordo com o sistema de classificação do grupo de estudo de uveíte internacional. A uveíte foi considerada como não infecciosa na ausência de evidências de infecção, após uma pesquisa diagnóstica. A uveíte foi classificada como crônica quando persistente por mais de três meses ou recorrente no período de três meses. Os pacientes usaram o metotrexate com doses iniciais de $7,5 \mathrm{mg}$ por semana, junto com ácido fólico $1 \mathrm{mg}$ ao dia. Alguns pacientes que não apresentaram resposta ao metotrexate utilizaram a sua associação com ciclosporina, a uma dose inicial desta de $2,5 \mathrm{mg} \mathrm{kg} / \mathrm{dia}$ (geralmente 150 a $200 \mathrm{mg} / \mathrm{dia}$ ). Dos 160 pacientes estudados, $74 \%$ eram mulheres com idade média de início da uveíte de 33 anos. A duração média da uveíte antes do início do metotrexate foi 5 anos e 3 meses. O tempo médio de tratamento com metotrexate foi de 16,4 meses. Com relação ao tipo de uveíte, $2 / 3$ tinham uveíte anterior, $15 \%$ dos casos tinham panuveíte e $20 \%$ tinham uveíte intermediária e/ou posterior. Uma doença sistêmica relacionada com a uveíte estava presente em $64 \%$ dos pacientes tratados. Entre as causas de uveíte, $37 \%$ apresentavam uveíte HLA-B27 positiva, $21 \%$ artrite reumatóide juvenil, 10\% sarcoidose, 9\% espondiloartropatia soronegativa HLA-B27 negativa e 5\% doença inflamatória intestinal, sendo as outras patologias sistêmicas menos freqüentes. A dose do metotrexate variou de 7,5 a $40 \mathrm{mg}(12,3 \mathrm{mg} / \mathrm{semana}$ em média). Dos 14 pacientes que utilizaram a combinação de metotrexate e ciclosporina, $11(71 \%)$ melhoraram. Os autores entendem que o metotrexate pode ser utilizado de forma segura nos pacientes com uveíte, mas alertam que a natureza retrospectiva do estudo atual justifica uma interpretação ainda cuidadosa dos resultados. Os autores concluem nesta série de casos publicada (a maior até o momento), que o uso do metotrexate em uveítes é efetivo nos pacientes não responsivos ao uso de corticosteróides e também nos pacientes que tiveram efeitos colaterais com os corticosteróides. 
Sfikakis PP, Kalamanis P, et al: Successful treatment of ocular relapse in patients with Behcet's disease with a single infusion of the anti-TNF agent Infliximab (Tratamento eficaz das recidivas oculares nos pacientes com doença de Behçet). Arthritis 46: S181, Abstract 399, 2002. Instituição: Hospital geral da Universidade de Athenas, Grécia.

Uma infusão isolada do anticorpo monoclonal anti-TNF infliximab foi dada a 5 pacientes com doença de Behçet e panuveíte grave com risco de perda de visão. A terapêutica imunossupressora anterior foi mantida nas mesmas doses ou foi aumentada nos 5 pacientes. Houve melhora, com supressão efetiva da inflamação ocular naqueles pacientes, o que foi publicado no Lancet de 2001. Os autores estenderam esta investigação, agora com 14 pacientes com doença de Behçet, mas com o uso de infliximab como monoterapia. Uma infusão única de infliximab de $5 \mathrm{mg} / \mathrm{kg}$ foi administrada a 10 homens e 4 mulheres, com idades variando entre $21 \mathrm{e}$ 56 anos. Os pacientes tinham história de múltiplas recorrências oculares, tendo a última ocorrido nas últimas 48 horas da administração do infliximab. O infliximab foi administrado como tratamento inicial em 11 olhos de pacientes não tratados, ou associados ao tratamento anterior em 13 olhos. Exames foram realizados para avaliação no dia 1, 7, 14 e 28 após o tratamento. Recorrências oculares consistiram de uveíte anterior em 3 olhos, uveíte posterior em 12 olhos e panuveíte em 9 olhos, com ou sem vasculite de retina (17 e 7 olhos, respectivamente). A acuidade visual estava reduzida em todos os pacientes, porém, no primeiro dia após o início do tratamento, houve melhora significante da acuidade visual, forte redução das células na câmara anterior e redução também na turvação do vítreo (média 50\%). Inflamação ocular aguda incluindo vasculite e lesões da retina resolveram completamente em 7 dias (12 olhos, incluindo 1 com hipópio) ou em 14 dias (completamente em 7 olhos e em $80 \%$ a $90 \%$ em 5 olhos). A acuidade visual retornou aos níveis anteriores ao episódio inflamatório atual (última recidiva) em todos os pacientes. Edema macular cistóide, presente em 5 pacientes, melhorou de forma significante no dia 28 em 3 olhos e permaneceu estável em 2 olhos. As lesões da retina crônica não sofreram influência do uso do infliximab. Os pacientes que tinham oligoartrite ( 5 pacientes ) e os pacientes que tinham aftas orais durante o estudo ( 7 pacientes ) tiveram melhora destes sintomas em 1 a 4 dias após a infusão. Não foram observados efeitos adversos em qualquer dos pacientes. Os autores assim propõem que a eficácia de uma única aplicação de infliximab constitui o tratamento de escolha nesta situação ocular grave.

Ylmaz O, Fresko I, Yasici $\mathrm{H}$, et al: The response of treatment resistant uveitis in Behçet's syndrome (BS) to a TNF $\alpha$ blocker, etarnecept: A open study (A resposta ao tratamento da uveíte refratária na síndrome de Behçet a um bloqueador do TNF $\alpha$ etanercept: um estudo aberto). Arthritis 46: S181, Abstract 400, 2002. Instituição: Centro de pesquisa em síndrome de Behçet, Faculdade de Medicina Cerrahpasa, Istanbul, Turquia.

O objetivo do estudo foi determinar a resposta ao tratamento da uveíte resistente ao tratamento na síndrome de Behçet, com o uso do bloqueador do TNF- $\alpha$ etanercept. Foram estudados 10 pacientes do sexo masculino, com idade média de 29,8 anos. Dentre os critérios de inclusão para o estudo, os autores exigiram que os pacientes tivessem tido pelo menos um ataque de panuveíte durante os últimos seis meses, uma acuidade visual de pelo menos $1 / 10$ na escala visual de Snellen para um dos olhos e refratariedade ao tratamento prévio com a combinação de corticosteróides, ciclosporina e azatioprina. Todos os pacientes receberam injeções subcutâneas de $25 \mathrm{mg}$ de etanercept 2 vezes por semana por um período total de 6 meses, além das medicações que os pacientes estavam previamente usando, exceto a ciclosporina A, que teve de ser descontinuada. Os pacientes foram, então, avaliados com intervalos mensais por um oftalmologista. O período de seguimento pós-tratamento foi de 53 meses e foi possível em todos os pacientes inicialmente tratados. As diferenças da acuidade visual com relação ao início do tratamento foram analisadas com teste $\mathrm{t}$ pareado. Os autores também avaliaram as doses de corticosteróides utilizadas, antes, durante e após o tratamento. A acuidade visual melhorou em 6 dos 10 pacientes, e permaneceu estável nos outros 4 pacientes. Após o término da terapia a acuidade visual voltou a piorar em 7 pacientes, permaneceu estável em 2 pacientes e melhorou em 1 paciente. Houve redução das doses de corticosteróide em 8 dos 10 pacientes durante o tratamento, enquanto o 
requerimento para corticosteróide aumentou após o período do tratamento em 7 pacientes. Os autores assim concluem que o efeito do etanercept foi benéfico em pelo menos manter a acuidade visual em um pequeno grupo de pacientes com doença de Behçet, mas este efeito não é mantido após o término do tratamento.

Rosenberg AM: Uveitis associated eith childhood rheumatic diseases (Uveíte associada com doenças reumáticas da infância). Curr Opin Rheumatol 14: 542 7, 2002. Instituição: Saskatoon, Canadá.

Aproximadamente 30\% a 40\% das crianças com uveíte têm uveíte anterior, $40 \%$ a $50 \%$ têm uveíte posterior, $10 \%$ a $20 \%$ têm uveíte intermediária e $5 \%$ a $10 \%$ tem panuveíte. A uveíte anterior assintomática crônica está nitidamente relacionada com a artrite idiopática juvenil oligoarticular e representa uma entidade distinta. A prevalência da uveíte associada à artrite idiopática juvenil tem sido menos freqüente atualmente, mas varia entre $6 \%$ e $17 \%$. O prognóstico da uveíte associada à artrite idiopática juvenil está melhorando, com menor incidência atual de perda de visão ou de outras complicações oculares, em razão do diagnóstico precoce, monitoração mais vigilante da doença ocular e terapia imunossupressora mais agressiva usada no tratamento da doença articular concomitante. As uveítes posteriores, embora bem menos freqüentes, também podem ocorrer na infầncia e são caracterizadas pela inflamação da retina, coróide, vítreo e corpo ciliar. As formas não infecciosas de uveíte posterior incluem aquelas causadas pela doença de Behçet, sarcoidose, Vogt-Koyanagi-Harada, oftalmia simpática, uveíte intermediária e corioretinopatia de birshot. Nefrite túbulo-intersticial e síndrome da uveíte, uma condição de início mais freqüente na infância e adolescência, parece ser mais comum atualmente do que se acreditava. A uveíte associada à nefrite túbulo-intersticial geralmente é anterior, mas ambas as formas intermediária e posterior podem ocorrer. Pars planitis, uma uveíte intermediária, representa entre $15 \%$ e $20 \%$ das crianças com uveíte e é considerada uma doença das crianças e adultos jovens. O início logo na infầncia parece estar associado a uma acuidade visual pior, tanto no início quanto no seguimento. $\mathrm{O}$ tratamento da uveíte não infecciosa continua sendo a corticoterapia tópica. Em razão da toxicidade potencial do corticosteróide a longo prazo, pesquisas continuam para o encontro de terapias mais efetivas e opções terapêuticas mais seguras. Estudos iniciais sugeriam o uso do clorambucil no tratamento da uveíte da artrite idiopática juvenil. A experiência com o uso do inibidor do fator de necrose tumoral alfa etanercept em 10 pacientes foi relatada no tratamento de crianças com uveíte refratária a corticosteróide tópico, metotrexate e ciclosporina. Sete dos pacientes tinham uveíte associada à artrite idiopática juvenil e 3 tinham uveíte idiopática. Dos 16 olhos avaliados, 10 (63\%) mostraram redução nos indicadores de inflamação ocular. Outro estudo, com inibidor de TNF em 16 pacientes, mostrou melhora da doença ocular em 38\%: este grupo incluiu 4 pacientes com uveíte associada à artrite idiopática juvenil. Num estudo prospectivo de 10 anos, Gerloni et al. relataram sua experiência no tratamento de 34 pacientes com artrite juvenil e ciclosporina, sendo que deste total 7 pacientes (21\%) tinham uveíte associada. Dos 12 olhos acometidos, 8 (66\%) mostraram melhora nos sinais de inflamação ocular. A acuidade visual melhorou em 7 dos 12 olhos acometidos (58\%) e uma redução da terapia com corticosteróide foi assim possível. Intervenção cirúrgica em alguns foi responsável pela melhora do quadro ocular. Seis dos 7 pacientes com uveíte tratados com ciclosporina desenvolveram efeitos adversos, incluindo aumento da creatinina (3 pacientes), hipertricose (2 pacientes) e hiperplasia gengival (2 pacientes). Com relação à terapia ocular futura, o uso de implante ocular intra-vítreo poderá contribuir para a melhora do tratamento.Apesar do reduzido número de pacientes, a gravidade do problema e a ótima resposta a esta nova terapêutica alertam para a sua utilização em situações de risco de perda de visão.

Ohara K: Ocular sarcoidosis (Sarcoidose ocular). Nippon Rinsho Japanese Journal of Clinical Medicine 60: 1807 12, 2002. Instituição: Escola médica de Nipon.

O envolvimento ocular na sarcoidose é freqüente e pode ser a manifestação clínica inicial da doença. As lesões ocu- lares mais comuns incluem uveíte granulomatosa associada a nódulos trabeculares na íris, faixas de opacidade no vítreo 
e perivasculite da retina, principalmente afetando veias e exsudatos na retina e coróide. Metade dos pacientes com lesões oculares típicas sugestivas de sarcoidose não mostraram evidências de comprometimento sistêmico, permanecendo com quadro de sarcoidose provável. Os riscos de deterioração visual são glaucoma secundário, opacidades no vítreo, edema macular cistóide e neovascularização da retina. Do total de pacientes, 34\% foram tratados com corticosteróide sistêmico, sendo que alguns deles requereram outros tratamentos, tais como o metotrexate. O porcentual dos que evoluíram com acuidade visual pobre foi de $21 \%$.

Castro ED, Cantalapiedra JMH, Cano CM, Osés S: Ocular sarcoidosis . Retrospective study of 18 cases (Sarcoidose ocular. Estudo retrospectivo de 18 casos). Arch Soc Espanhola de Oftalmol 77: 301 8, 2002. Instituição: Universidade de Valladolid, Espanha.

Os autores analisaram as características clínicas e epidemiológicas, os métodos diagnósticos e o prognóstico visual final de pacientes com sarcoidose ocular. Trata-se de um estudo retrospectivo de 18 pacientes, no período de março de 1989 a maio de 1999. Todos os pacientes tiveram os seguintes dados analisados: idade, sexo, bilateralidade, prognóstico visual inicial e final, manifestações ocular e sistêmica, estágio da radiografia do tórax, marcadores laboratoriais, cintilografia com gálio, resultado da biópsia, tratamento clínico e cirúrgico e complicações do tratamento. A idade média de início da doença foi 49,7 anos, 14 pacientes $(77,8 \%)$ eram mulheres e $4(22,2 \%)$ eram homens. O tempo médio de acompanhamento foi de 40,3 meses. Nove pacientes (50\%) foram submetidos à biópsia. A presença de granuloma não caseoso foi constatada em 7 $(77,8 \%)$. A cintilografia com gálio foi positiva em 16 pacientes
(88,8\%). A manifestação ocular mais comumente encontrada foi panuveíte (40\%). Entre os 30 olhos estudados, envolvimento conjuntival foi encontrado em 10 (33,3\%), catarata secundária em 9 (30\%) e glaucoma secundário em 6 (20\%). Onze pacientes $(61,1 \%)$ foram tratados com corticosteróide oral e $10(55,5 \%)$ com ciclosporina A. No momento em que a sarcoidose foi diagnosticada, 11 olhos (36,6\%) apresentaram acuidade visual melhor que 0,6 , e ao fim do seguimento a acuidade visual foi melhor que 0,6 em 17 olhos (56,6\%). Os autores assim concluem que o controle adequado da inflamação ocular pode melhorar o prognóstico nos pacientes com sarcoidose ocular e relatam que nestes pacientes o envolvimento do segmento posterior (uveíte posterior, edema macular ou membrana epitelial) pode estar associado a um pior prognóstico visual.

Kruithof E, Kestelyn P, Elewaut C, et al: Successful use of infliximab in a patient with treatment resistant spondyloarthropathy related uveitis (Uso eficaz do infliximab em um paciente com uveíte associado a espondiloartropatia resistente ao tratamento). Ann Rheum Dis 61: 470, 2002. Instituição: Hospital universitário de Ghent, Bélgica.

Uveíte anterior e intermediária são processos inflamatórios de causas diversas, que incluem trauma, doenças linfoproliferativas e isquemia. As outras etiologias das uveítes têm patogênese imune, com um componente autoimune associado. Uveíte é uma manifestação comum no grupo das espondiloartropatias e pode ocorrer em $40 \%$ dos pacientes. Nestes casos, a uveíte se caracteriza por ser anterior e aguda. Aliás, das causas de uveíte anterior aguda, as espondiloartropatias soronegativas são as doenças sistêmicas mais comuns. Além disso, como acontece nos pacientes com espondiloartropatia soronegativa, a inflamação intestinal subclínica também tem sido encontrado nos pacientes com uveíte anterior aguda isolada, mesmo sem evidências clínicas de espondiloartropatia. Os efeitos benéficos do bloqueio do fator de necrose tumoral $\alpha$ com infliximab estão bem estabelecidos na doença de Crohn e atualmente também no controle dos sintomas articulares dos pacientes com espondiloartropatia. Considerando os aspectos de associação das espondiloartropatias com as uveítes e os bons resultados terapêuticos do bloqueio do TNF neste grupo de doenças, os autores relatam os efeitos do bloqueio do TNF infliximab na uveíte resistente ao tratamento em um paciente com 
espondiloartropatia. Trata-se de um paciente do sexo masculino com espondiloartropatia soronegativa HLA-B27 positiva, que apresenta uveíte recorrente do olho esquerdo e que evoluiu com uveíte anterior crônica e intermediária. O paciente não teve resposta com corticosteróide tópico, injeções subconjuntivais e corticosteróides sistêmicos. Não houve melhora também com sulfassalasina e ciclosporina. O paciente foi então submetido a um esquema de indução com infliximab $5 \mathrm{mg} / \mathrm{kg}$ na semana 0 , semana 2 e semana 6. Após 1 semana da indução, a acuidade visual melhorou de forma marcante. Esta melhora foi mantida até a semana 14, ou seja, 8 semanas após a última infusão, após a qual o paciente teve recorrência. O retratamento com $5 \mathrm{mg} / \mathrm{kg}$ na semana 14 foi, contudo, novamente eficaz em melhorar a inflamação ocular e a acuidade visual. A partir de então o paciente foi mantido em tratamento a cada 8 semanas, sendo que a inflamação permaneceu estável. Não houve necessidade futura de injeções subconjuntivais ou de corticosteróides sistêmicos durante este período. A dose de corticosteróide oral foi substancialmente reduzida. Considerando os efeitos benéficos do infliximab na doença de Crohn e nas espondiloartropatias e a observação da eficácia clínica neste paciente com uveíte relacionada com espondiloartropatia, os autores sugerem a exploração deste potencial terapêutico nas uveítes relacionadas com espondiloartropatia soronegativa. 